\title{
Distribution et autoécologie de l'écrevisse à pattes blanches, Austropotamobius pallipes (Ler.) à Bizkaia (Pays Basque, Espagne)
}

\author{
B. G. de Bikuna 1 \\ L. Docampo ${ }^{2}$ \\ R. Asensiol
}

Mots clés : Crustacea, Austropotamobius pallipes, Procambarus clarkii, autoécologie, étang.

Ce travail examine la distribution d'Autropotamobius pallipes (Ler.) et des caractéristiques abiotiques des écosystèmes aquatiques que cette espèce colonise en Bizkaia. 81 stations du réseau hydrographique de Bizkaia et 17 étangs situés dans une zone minière près de Bilbao ont été échantillonnés et leurs eaux analysées.

On constate d'une part, la disparition de l'espece dans la plupart des rivières et d'autre part, sa grande abondance dans les étangs. L'analyse des spectres écologiques permet de déterminer l'affinité écologique de l'écrevisse pour les eaux froides et dures et sa tolèrance à l'eutrophisation.

Distribution and autoecology of the crayfish Austropotamobius pallipes Ler. in Bizkaia (Basque Country, Spain)

Keywords : Crustacea, Austropotamobius pallipes, Procambarus clarkii, autoeccology, pond.

This work examines the distribution of $A$. pallipes and the abiotic characteristics of the aquatic ecosystems this species colonizes in Bizkaia.

Waters from 81 sampling sites in the hydrographic network of Bizkaia were analysed, and 17 ponds in a mining area surrounding Bilbao were studied. This species has disappeared from most rivers but is still abundant - in the pools.

Range habitat analysis enables us to assess the affinity of this species to cold and hard waters and its tolerance to eutrophication.

\section{Introduction}

En 1968, l'espèce Austropotamobius papilles (Lereboullet 1858) était considérée comme très courante au Portugal et dans 26 provinces espagnoles (Zariquiey 1968) (fig. 1) ; depuis, à cause de l'aphanomycose, les populations d'écrevisses ont nettement régressé.

Les mortaiités massives ont été constatées dans la rivière Duero vers la fin des années 50 et dans la rivière Ucero (Soria) dans les années 60 . Actuellement, cette espèce vit dans certaines rivières où la

1. Departamento de Biologia Vegetal y Ecologia. Facultad de Ciencias. Universidad del País Vasco-E.H.U. Apdo : 644 (48080) Bilbao, Espagne.

2. Departamento de Biología Animal y Genética. Faculiad de Ciencias. Universidad del País Vasco - E.H.U. Apdo : 644 (48080) Bilbao, Espagne. température ne favorise pas l'apparition de la peste, bien que la distribution des dernières populations soit mal connue (Zariquiey 1968). Actuellement, plusieurs études se sont développées, principalement dans le Nord-Ouest de la péninsule Ibérique, pour connaître la situation astacicole. Des inventaires de populations ainsi que des études des caractéristiques écologiques de ses habitats, et du repeuplement systématique à grande échelle avec des espèces adéquates - Austropotamobius pallipes et Pacifastacus leniusculus - sont ef fect uées (Celada et al. 1984, 1986 ; Celada \& Gaudioso 1985 ; Temiño et al 1986).

La peste provoquée par Aphanomyces astaci, apparue au début de l'été 1978, a été la cause de la disparition quasi-totale de l'espèce dans les rivières basques (Salazar 1983). Actuellement il existe un intérêt croissant pour l'étude de l'écrevisse. Aussi l'inventaire des populations qui sont restées dans nos 


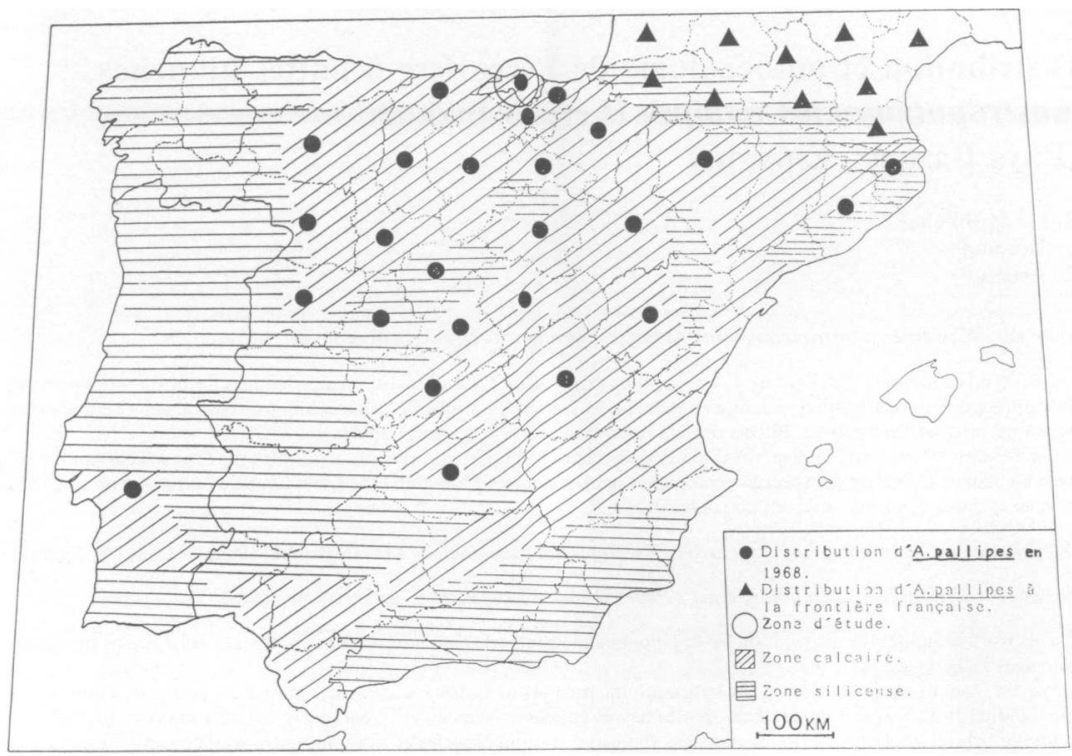

Fig. 1. Distribution d' $A$. pallipes dans la Péninsule lbérique en 1968.

rivières et l'étude des caractéristiques écologiques de ses habitats constituent des données fondamentales pour de futures études ainsi qu'un bilan des connaissances sur la distribution et l'autoécologie de l'espèce dans le Pays Basque.

\section{Localisation du site}

81 stations réparties dans 20 rivières principales appartenant à 13 bassins du réseau hydrographique de Bizkaia (Carranza, Agüera, Mercadillo, Galindo, Cadagua, Nervión, Ibaizabal, Artibai, Lea, Oka, Butrón, Asua et Gobelas) ont été échantillonnées (fig. 2 a).

L'étude morphométrique et typologique de ces bassins ainsi que la qualité physico-chimique et biologique de leurs eaux, a fait l'objet de plusieurs publications (G. de Bikuña 1987, G. de Bikuña et al., 1987, Docampo \& Rallo 1987).
Les rivières sont courtes, peu profondes, de flux rapide, avec des substrats, en grande partie, de sable et de graviers. Les parties moyennes et basses de ces rivières sont contaminées par des déversements urbains et industriels, les parties hautes, les petits ruisseaux, ainsi que ceux qui traversent les zones rurales, sont les seuls à présenter des conditions naturelles.

La moyenne des précipitations (annuelle) est de $1200 \mathrm{~mm}$ et la température de l'eau varie de 6 à $8^{\circ} \mathrm{C}$ en hiver et de 17 à $20^{\circ} \mathrm{C}$ en été.

12 étangs qui forment le système lénitique de la zone minière proche de Bilbao ont été échantillonnés. Les étangs $\mathrm{H}_{1}, \mathrm{H}_{2}, \mathrm{H}_{3}, \mathrm{H}_{5}, \mathrm{H}_{6}, \mathrm{H}_{7}$ et $\mathrm{H}_{9}$ se trouvent sur des versants peu prononcés, à 200-300 m d'altitude, et les étangs $\mathbf{H}_{4}, \mathbf{H}_{10}, \mathrm{H}_{11}$ et $\mathrm{H}_{12}$ sont situés sur des versants plus prononcés à $375-475 \mathrm{~m}$ d'altitude (fig. 2 b). Le substrat géologique est constitué de calcaires du Crétacé inférieur. 
Ceci détermine en grande partie les caractéristiques physico-chimiques des eaux. La végétation riveraine des rivières est formée par du Salix caprea et du Salix $a l b a$, qui constituent une ceinture périphérique. Les espèces Thypha latifolia, Nasturtium officinale, Equisetum sp et Juncus sp. se situent à moins d' $1 \mathrm{~m}$. Le fond des étangs est recouvert d'algues, Chara sp.

Ces étangs proviennent d'anciennes mines, où l'on trouve actuellement une structure écologique d'une grande maturité, avec des caractéristiques d'oligotrophie en hiver et de mésotrophie en été pour ceux dont le diamètre est. supérieur à $50 \mathrm{~m}$ et la profondeur supérieure à $10 \mathrm{~m}$, et une forte eutrophie en été dans les petits marécages.

\section{Méthodes d'études}

L'étude des étangs a été réalisée en 1982 et celle des rivières en 1985. Quatre échantillonnages, chacun à une saison différente : hiver, printemps, été et automne, ont été effectués. A chaque saison on a pris des échantillons d'eau afin de déterminer le degré de minéralisation et d'eutrophie des habitats.
Le $\mathrm{pH}$, la température, l' $\mathrm{O}_{2}$ dissous et la conductivité ont été mesurés « in situ». Les chlorures, le sodium, le potassium, les nitrites, les nitrates, l'ammonium, la dureté totale, le calcium, le magnésium, l'alcalinité totale, les sulphates, les carbonates, les bicarbonates, les orthophosphates et la matière organique (M.O.) ont été mesurés au laboratoire, d'après les techniques d'analyses du APHA-AWWA-WPCF (1981) et Golterman (1969).

Pour la capture d'écrevisses nous avons essentiellement utilisé trois modes de pêche, employés normalement pour des études semblables (Roqueplo 1983), mais avec un caractère qualitatif, non quantitatif :

- Pêche de nuit à la main, en remontant le ruisseau ;

- Pose de nasses appâtées pendant la nuit et le jour ;

- Pêche électrique pendant le jour.

Pour obtenir une description des conditions abiotiques, une analyse en composantes principales, selon le système statistique B.M.D.P. (BMDP

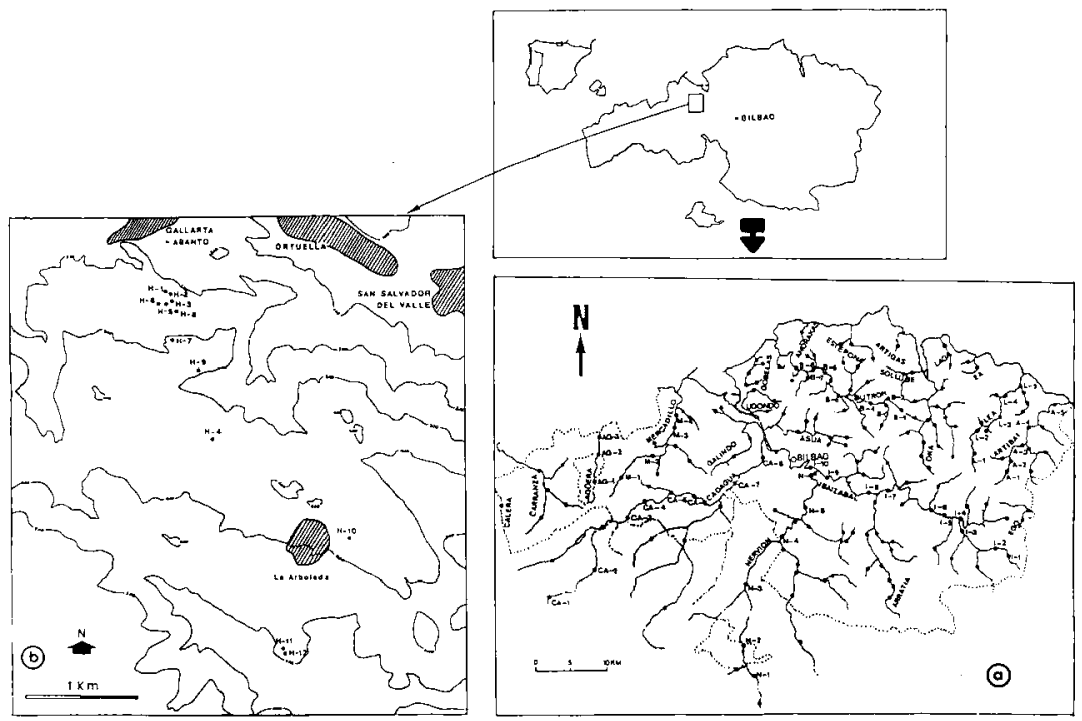

Fig. 2. Zone d'études : a) Stations échantillonnées dans les rivières. b) Localisation des étangs étudiés. 
Statistical Software Inc. 1984) a été réalisée. Les données ont été normalisées par la transformation $\log \mathrm{x}+1$.

\section{Résultats et discussion}

\subsection{Rivières}

L'eau des rivières de Bizkaia présente des caractéristiques physico-chimiques propres à une zone calcaire avec prédominance du bicarbonate calcique : $\mathrm{HCO}_{3}$ - représente $73,10 \%$ du contenu total en anions, $\mathrm{CA}++60,20 \%$ du contenu total en cations (fig. 3).

Sur le tableau I on peut voir les caractéristiques des cinq groupes de bassins statistiquement différents en fonction de la minéralisation, (G. de Bikuña, données non publiées). Les écrevisses ont été trouvées principalement dans les ruisseaux des bassins appartenant aux groupes de minéralisation 2 et 3.

Dans le but d'obtenir une vision synthétique de l'habitat hydrochimique, on a réalisé une analyse en composantes principales (A.C.P.). Le tableau II présente les corrélations des variables sur les trois premiers axes. Ces trois axes expriment respectivement
$42,4,17,2$ et $13 \%$ de la variance. La position des variables sur plan est donnée par le degré de corrélation par rapport aux trois axes.

Les variables les plus corrélées sur l'axe I sont, dans le sens positif de l'axe, la conductivité, les chlorures, la dureté, le calcium et les sulfates. Les phosphates aussi sont corrélés, mais à un degré moindre. En opposition à ces variables se trouvent;l'oxygène dissous et le pourcentage de saturation d'oxy-

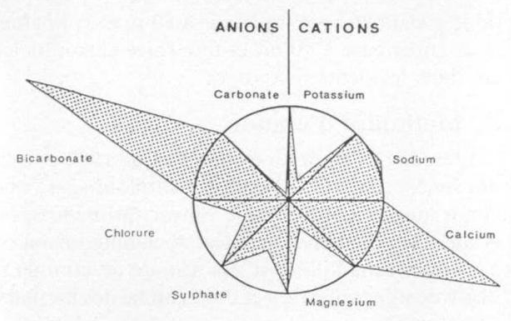

Fig. 3. Diagramme de proportionnalité ionique pour les rivières étudiées.

Tableau I. Principales caractéristiques physico-chimiques des rivières étudiées. On indique la valeur moyenne $(\overline{\mathrm{X}})$, maxima $(\overline{\mathrm{X}} \mathrm{max})$ et minima $(\overline{\mathrm{X}} \min )$ des moyennes $(\overline{\mathrm{X}} \max =\overline{\mathrm{X}}+\mathrm{sR} ; \overline{\mathrm{X}} \min =\overline{\mathrm{X}}-\mathrm{sR}) ;(\mathrm{Sr}=$ erreur standard $)$.

\begin{tabular}{|c|c|c|c|c|c|c|c|}
\hline $\begin{array}{l}\text { GROUPES DE } \\
\text { MINERALISATION }\end{array}$ & BASSINS & & $\begin{array}{l}\text { CONDUCTIVITE } \\
\mu \mathrm{S} . \mathrm{cm}^{-1}\left(25^{\circ} \mathrm{C}\right)\end{array}$ & $\begin{array}{l}\text { ALCALINITE } \\
\text { meq. } 1^{-1}\end{array}$ & $\mathrm{pH}$ & $\begin{array}{l}\text { DURETE } \\
\mathrm{mg} \cdot \mathrm{1}^{-1}\end{array}$ & 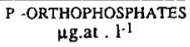 \\
\hline$x$ & $\begin{array}{l}\text { CARRANZA } \\
\text { AGÜERA }\end{array}$ & $\begin{array}{l}\overline{\bar{x}} \overline{\min }_{\overline{\mathrm{X}}} \\
\overline{\mathrm{X}} \max \end{array}$ & $\begin{array}{l}136 \\
146 \\
156\end{array}$ & $\begin{array}{l}0.89 \\
0,9 \\
0 ' 91\end{array}$ & $\begin{array}{l}7 \cdot 5 \\
7.6 \\
7.7\end{array}$ & $\begin{array}{l}76 \\
82^{\prime} 6 \\
88\end{array}$ & $\begin{array}{l}0.9 \\
1.1 \\
1 \cdot 3\end{array}$ \\
\hline II & $\begin{array}{c}\text { IBAIZABAL } \\
\text { ARTIBAI } \\
\text { LEA }\end{array}$ & $\begin{array}{l}\bar{x} \min \\
\bar{x} \\
\bar{x} \max \end{array}$ & $\begin{array}{l}293 \\
302 \\
311\end{array}$ & $\begin{array}{l}2.35 \\
2.43 \\
2 ' 51\end{array}$ & $\begin{array}{l}7 \% \\
7 \% \\
78\end{array}$ & $\begin{array}{l}149 \\
152 \\
155\end{array}$ & $\begin{array}{l}3 \cdot 3 \\
3 \cdot 8 \\
4 \cdot 3\end{array}$ \\
\hline$*$ & $\begin{array}{l}\text { MERCADILLO } \\
\text { NERVION } \\
\text { OKA } \\
\text { CADAGUA* } \\
\text { (zone inféricure) }\end{array}$ & $\begin{array}{l}\bar{x}{ }_{\bar{x}} \\
\bar{x}{ }_{\max }\end{array}$ & $\begin{array}{l}377 \\
395 \\
417\end{array}$ & $\begin{array}{l}26 \\
271 \\
28\end{array}$ & $\begin{array}{l}7.9 \\
8.1 \\
8.1\end{array}$ & $\begin{array}{l}182 \\
187 \\
193\end{array}$ & $\begin{array}{l}1.67 \\
2.17 \\
2.67\end{array}$ \\
\hline IV & $\begin{array}{l}\text { BUTRON } \\
\text { ASUA } \\
\text { GALINDO } \\
\text { GOBELAS }\end{array}$ & $\begin{array}{l}\bar{x} \min ^{2} \\
\bar{x} \text { miax }\end{array}$ & $\begin{array}{l}457 \\
533 \\
566\end{array}$ & $\begin{array}{l}35 \\
37 \\
34\end{array}$ & $\begin{array}{l}7 \% \\
76 \\
79\end{array}$ & $\begin{array}{l}20: \\
210 \\
217\end{array}$ & $\begin{array}{l}10 \\
135 \\
16\end{array}$ \\
\hline ** & $\begin{array}{l}\text { CADAGUA ** } \\
\text { (zone supćricure) }\end{array}$ & $\begin{array}{l}\bar{x} \min _{\bar{x}} \\
\bar{x} \max \end{array}$ & $\begin{array}{l}627 \\
658 \\
689\end{array}$ & $\begin{array}{l}37 \\
3 \times 3 \\
393\end{array}$ & $\begin{array}{l}800 \\
811 \\
812\end{array}$ & $\begin{array}{l}331 \\
358 \\
385\end{array}$ & $\begin{array}{l}2 \\
2 \cdot 8 \\
3.6\end{array}$ \\
\hline
\end{tabular}


Tableau 11. Coefficients de correlation entre chaque variable et les trois premiers axes de l'A.C.P.

\begin{tabular}{|c|c|c|c|}
\hline \multirow[b]{2}{*}{ VARIABLES } & \multirow[b]{2}{*}{ I } & \multicolumn{2}{|c|}{ COMPOSANTES } \\
\hline & & II & III \\
\hline $\mathrm{pH}$ & -0.27 & 0,137 & 0.286 \\
\hline$T^{\circ}$ & $-0,088$ & -0.116 & 0,399 \\
\hline conductivito & 0,535 & 0.274 & 0.040 \\
\hline$a_{2}$ & $-0,388$ & 0,358 & 0.124 \\
\hline$\times \mathrm{O}_{2}$ & $-0,375$ & 0,327 & 0,183 \\
\hline olcolinité & 0,308 & $-0,140$ & 0,171 \\
\hline dureté & 0,596 & 0,241 & 0.115 \\
\hline $\mathrm{Co}^{++}$ & 0,576 & 0.265 & 0,115 \\
\hline $\mathrm{Mg}^{++}$ & 0.590 & 0.100 & 0.073 \\
\hline $\mathrm{Cl}^{-}$ & 0.470 & 0,286 & 0,028 \\
\hline $\mathrm{SO}_{4}^{-}$ & 0.644 & 0,271 & 0.024 \\
\hline $\mathrm{PO}_{4}^{-3}$ & 0,374 & $-0,378$ & 0,044 \\
\hline $\mathrm{NO}_{2}^{-}$ & 0.198 & $-0,283$ & 0,067 \\
\hline $\mathrm{NO}_{3}^{-}$ & 0,114 & $-0,114$ & 0,147 \\
\hline $\mathrm{NH}_{4}^{+}$ & 0,278 & $-0,217$ & $-0,124$ \\
\hline $\mathrm{SiO}_{2}$ & $-0,137$ & 0,025 & 0,232 \\
\hline oltitude & $-0,010$ & 0,304 & $-0,433$ \\
\hline Dente & -0.135 & 0.136 & $-0,364$ \\
\hline
\end{tabular}

gène. Cet axe marque le degré de minéralisation de l'eau sans relation claire avec un gradient longitudinal source-embouchure qui, par contre, semble être mieux corrélé avec l'axe III. L'axe II montre le degré d'oxygénation de l'eau; l'oxygène dissous et le pourcentage de saturation d'oxygène s'opposent sur cet axe aux teneurs en phosphates, en ammonium, en nitrites et en nitrates. On peut trouver des données plus complètes sur les caractéristiques physico-chimiques des rivières étudiées dans $\mathrm{G}$. de Bikuña et al (1987) et Orive et al (1988). Les figures 4 et 5 situent dans l'ordre, les stations d'échantillonnage selon les résultats de l'A.C.P.

Sur la figure 4 on peut voir que les localités avec écrevisses se situent dans une zone d'oxygénation moyenne à haute avec une nette séparation entre les stations les plus oxygénées qui conservent l'espèce autochtone $A$. pallipes et les stations qui possèdent $P$. clarkii, dont le degré d'oxygénétion est plus bas. En ce qui concerne le degré de minéralisation, celuici ne fait pas de différence entre les stations où on a constaté la présence ou l'absence d'écrevisses puisque les eaux fluviales de Bizkaia présentent toujours un degré de minéralisation élevé (G. de Bikuña et al 1987).
Par rapport à la position des stations tout le long de l'axe III, celles qui ont des écrevisses se situent de préférence dans le quadrant inférieur positif de l'A.C.P. où prédominent les stations avec une plus grande altitude, une forte pente et des eaux froides et bien oxygénées.

Si l'on tient compte que l'échantillonnage porte sur des secteurs très étendus de rivières, ces stations ne correspondent pas à des points particuliers, mais à des zones où la présence d'écrevisses a été vérifiée. Dans quelques ruisseaux appartenant aux bassins de Agüera, Mercadillo, Gobelas, Butrón, Lea, Artibai et Arratia on a trouvé l'espèce Austropatamobius pallipes alors que dans les petits secteurs des bassins du Nervión, Asua et Galindo on a constaté la présence de l'espèce Procambarus clarkii (fig. 6).

Ces stations se trouvent surtout près des sources des rivières, situées à une altitude comprise entre $40 \mathrm{~m}$ (Gobelas) et $240 \mathrm{~m}$ (Arratia), où les populations sont peu nombreuses et sont restées isolées de la pollution et de l'aphanomycose.

Les plus grandes densités d'écrevisses autochtones se trouvent dans la rivière Castaño, affluent du Mercadillo. Dans les autres zones, les populations tendent à disparaître. Dans les conditions naturelles, $A$. pallipes occuperait une zone caractéristique de rivières appelée « zone à truite », représentée en Bizkaia par toutes les zones situées à plus de $200 \mathrm{~m}$ d'altitude et avec une pente de 2 à $10 \%$ (Docampo \& Rallo 1987). Le spectre écologique de l'espèce déterminé pour les écosystèmes fluviaux ( fig. 7) est plus large et plus fluctuant que celui qu'on retrouve pour les systèmes lénitiques; il présente des valeurs plus extrêmes que celles de la bibliographie ; habituellement on cite des valeurs de $\mathrm{pH}$ entre 5 et 7 '9 (Daguerre de Hureaux \& Roqueplo 1981 ; Roqueplo 1983), tandis que dans cette étude, on a trouvé des écrevisses dans des zones soumises à une pollution moyenne, avec un $\mathrm{pH}$ de 9 et une alcalinité de l'ordre de 9 à 10 meq. $\mathrm{HCO}_{3}^{-.} .^{-1}$ (groupe IV de minéralisation).

En ce qui concerne les paramètres liés à la minéralisation, on trouve l'écrevisse $A$. pallipes aussi bien dans des eaux qui présentent une faible minéralisation, avec une teneur en $\mathrm{Ca}^{2}+$ de $24 \mathrm{mg}^{-1}$ et une dureté totale de $72 \mathrm{mg}^{-1}{ }^{-1}$ de $\mathrm{CaCO}_{3}$ (dû en partie aux fortes teneurs en $\mathrm{Mg}^{2}{ }^{+}$), que dans les eaux très dures, avec des valeurs du $\mathrm{Ca}^{2}+$ de $104 \mathrm{mg.l}^{-1}$ et 


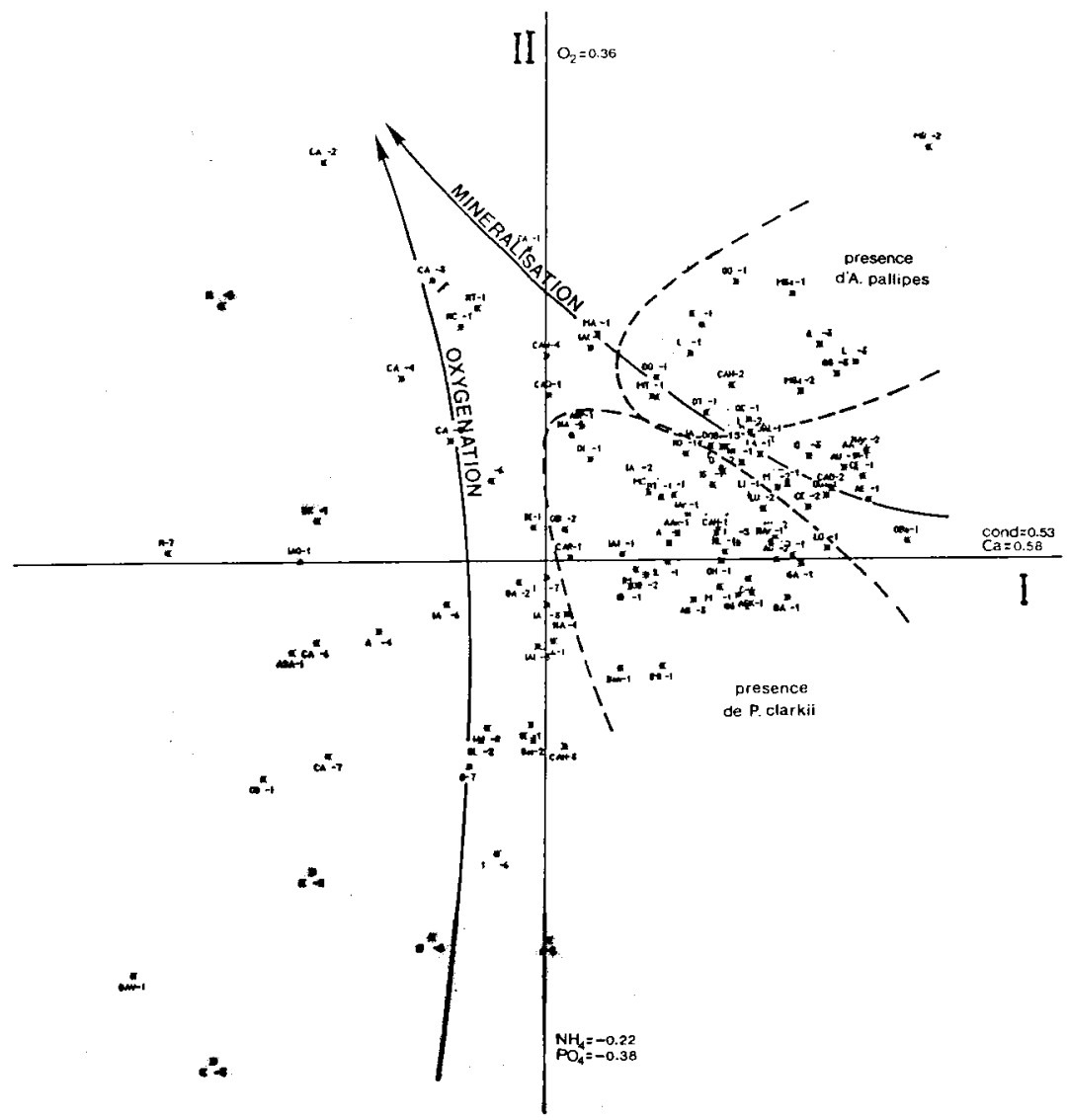

Fig. 4. Position des stations d'échantillonnage sur le plan établi entre les axes I et II de l'A.C.P. 


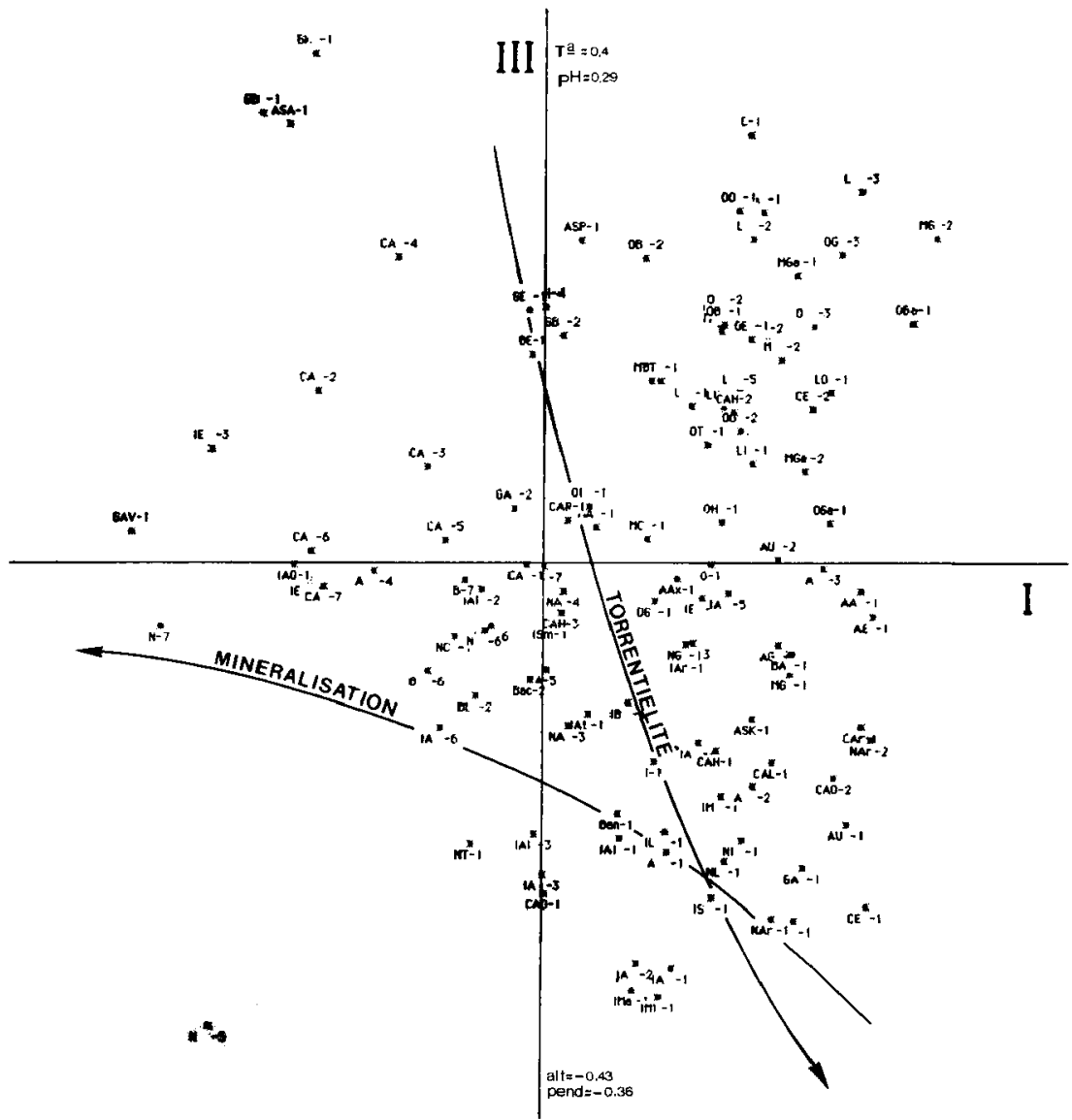

Fig. 5. Position des stations d'échantillonnage sur le plan établi entre les axes I et III de l'A.C.P. 


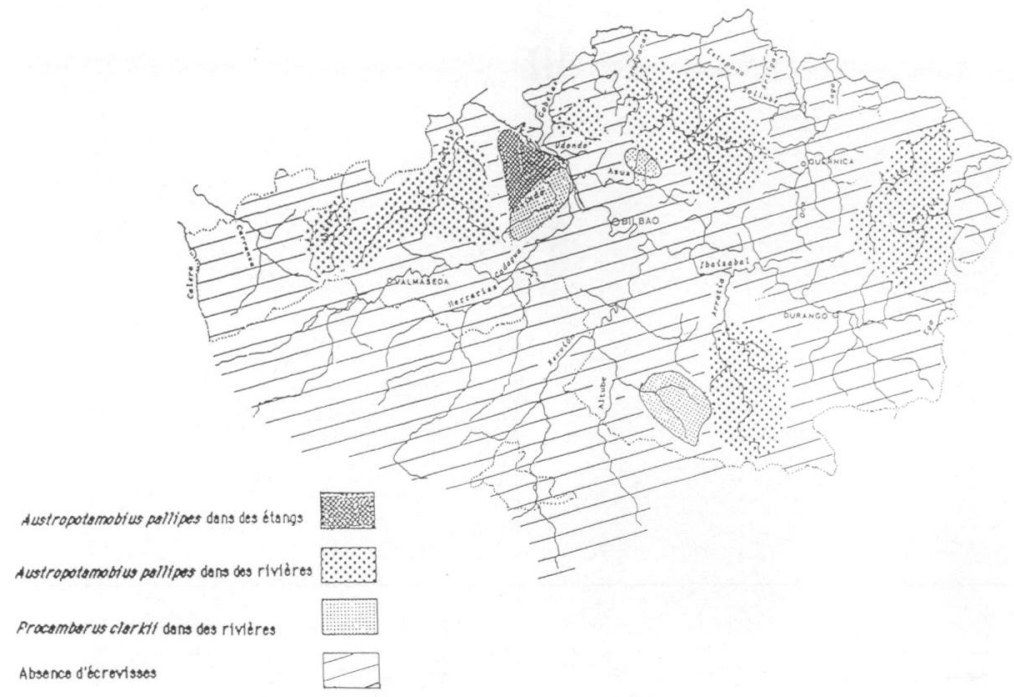

Fig. 6. Distribution d'A. pallipes et $P$. clarkii dans les rivières et étangs de Bizkaia (année 1985).

460 mg.l-1 de $\mathrm{CaCO}_{3}$, bien que la plupart se situent à des niveaux de dureté moyenne.

L'écrevisse autochtone supporte une certaine eutrophie puisqu'on l'a trouvée dans des eaux avec une teneur en phosphates de 0,3 à $54 \mu$ g.at. $1^{-1}$ de P- $\mathrm{PO}_{4}{ }^{-3}$ et une teneur en nitrites de 0 à $1,30 \mathrm{mg} . \mathrm{I}^{-1}$ de $\mathrm{NO}_{2}$. Cette dernière valeur est très élevée car la teneur moyenne se situe aux environs de $0,10 \mathrm{mg}$. $\mathrm{l}^{-1}$. La teneur en sulfates varie entre $13 \mathrm{mg} \mathrm{I}^{-1}$ et $39 \mathrm{mg} . \mathrm{I}^{-1}$.

L'espèce $A$. pallipes a une préférence pour les eaux froides avec une température moyenne annuelle de $12,5^{\circ} \mathrm{C}$, des températures maxima de $18^{\circ} \mathrm{C}$ en été et minima de $5^{\circ} \mathrm{C}$ en saison hivernale. Ces valeurs coïncident avec celles données par Laurent (1980), qui donne un niveau de tolérance de $21^{\circ} \mathrm{C}$.
On trouve cette espèce surtout dans les eaux bien oxygénées, avec des teneurs en $\mathrm{O}_{2}$ proches de $10 \mathrm{mg} . \mathrm{l}^{1}$, sursaturées dans la plupart des cas, bien que les valeurs supportées varient entre 6 et $15 \mathrm{mg}$. $\mathrm{I}^{-1}$.

L'espèce Procambarus clarkii a été récoltée dans les trois bassins fluviaux à plus forte densité humaine : Nervión ( $\left.94 \mathrm{hab} / \mathrm{km}^{2}\right)$, Galindo (4 $336 \mathrm{hab} / \mathrm{km}^{2}$ ) et Asua $\left(250 \mathrm{hab} / \mathrm{km}^{2}\right)$. Cette espèce a déplacé les $A$. pallipes de ces zones oủ autrefois elle était très nombreuse et connue des habitants de la région.

\subsection{Etangs}

On a trouvé des $A$. pallipes dans les 15 étangs permanents échantillonnés. L'origine de l'écrevisse y remonte à deux décennies. Elle a été introduite prin- 


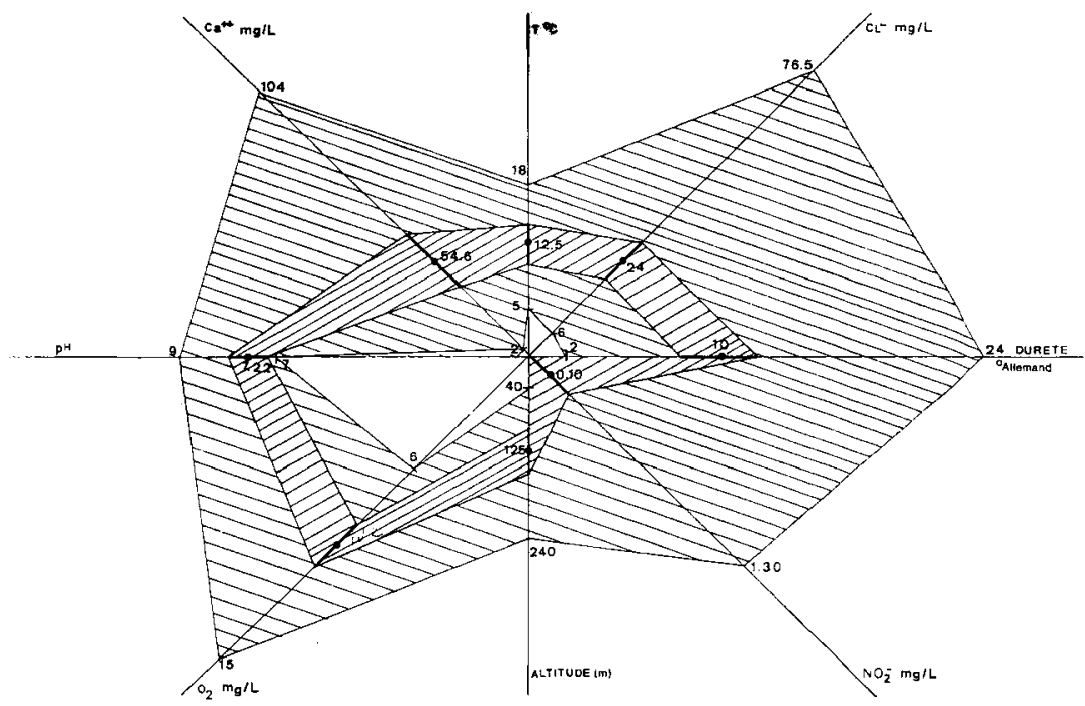

Fig. 7. Spectre écologique de l'espèce $A$. pallipes dans les rivières. On indique les valeurs moyennes, minimales et maximales, et l'intervalle de confiance à $95 \%$ pour chaque variable physico-chimique. La dureté est exprimée en degrés allemands $\left(1{ }^{\circ} \mathrm{dH}=171.9\right.$ mg $\left.\mathrm{CaCO}_{3} / 1\right)$.

cipalement par les émigrants mineurs provenant d'autres provinces.

Les populations de ces étangs sont restées à leur état naturel et n'ont pas été touchées ni par la pollution ni par les maladies, qui, par contre, ont décimé l'espèce dans le reste du territoire. Ces populations sont assez importantes, en 1982 on a compté de 100 exemplaires dans les petits étangs, à 1000 dans les étangs plus grands.

Zariquiey (1968) situe la ponte de cette espèce au mois de mars, par contre, nous avons pu observer dans les étangs, des femelles ovigères aux mois de novembre et décembre.

Le spectre écologique de l'espèce dans les étangs est représenté sur la figure 8 . Comme on l'a dit pré- cédemment, il est plus strict que celui trouvé dans les rivières: ceci peut être dû au fait que la diversité des milieux est plus élevée dans ces rivières que dans les étangs qui ont tous la même origine et le même substrat géologique.

L'espèce se trouve dans des étangs avec des températures inférieures à celles des rivières, mais les amplitudes supportées sont similaires $\left(6-20^{\circ} \mathrm{C}\right)$. En général tous les paramètres présentent une marge de variation plus étroite, bien que les différences ne soient pas excessives. Ainsi, en dureté on ne dépasse jamais le seuil de $300 \mathrm{mg}$. $\mathrm{l}^{-1}$ de $\mathrm{CaCO}_{3}$, de même, la valeur des chlorures la plus élevée est de $64 \mathrm{mg}$. $1^{-1}$. La valeur, la plus élevée des nitrites se situe aux environs de $0.33 \mathrm{mg} .1^{-1}$, sensiblement plus basse que celle obtenue dans les rivières. 


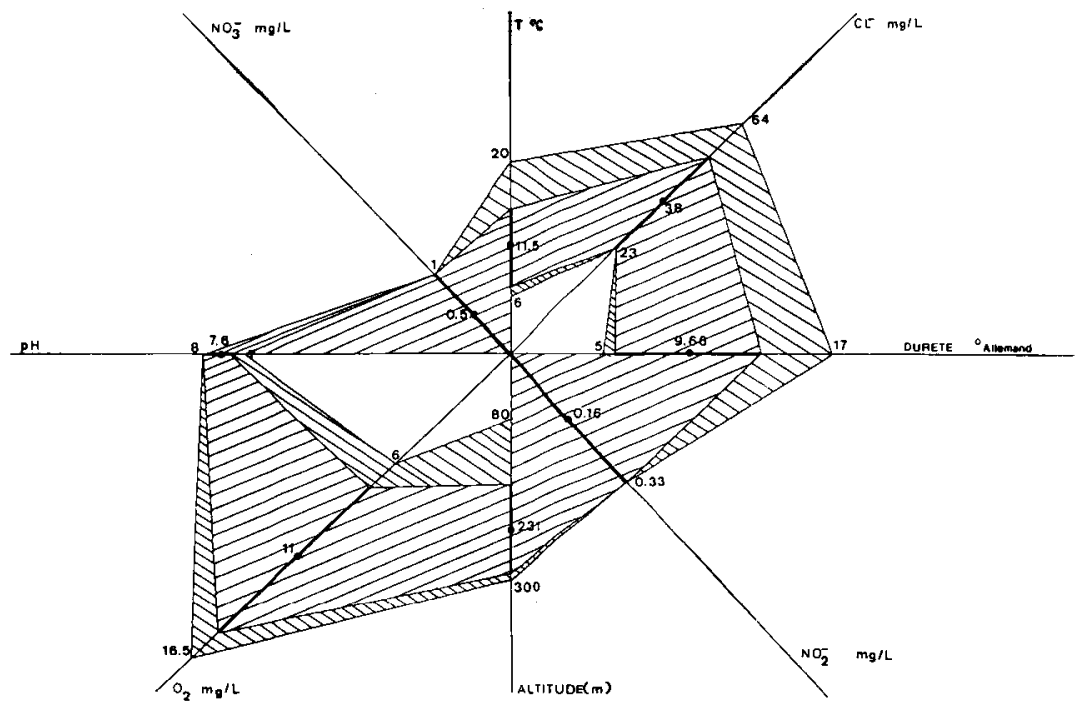

Fig. 8. Spectre écologique de l'espéce $A$. pallipes dans les étangs. On indique les valeurs moyennes, minimales et maximales, et l'intervalle de confiance à $95 \%$ pour chaque variable physico-chimiquei. La dureté est exprimée en degrés allemands $\left(1^{\circ} \mathrm{dH}=17.9 \mathrm{mg}\right.$ $\mathrm{CaCO}_{3} / \mathrm{l}$.

\section{Conclusion}

En Bizkaia, l'A. pallipes a été localisée dans deux écosystèmes aquatiques différents : les rivières et les étangs. Dans les rivières, la pollution, les épidémies et l'introduction des espèces allochtones ( $P$. clarkii) ont supprimé l'espèce autochtone de la plupart du territoire. Des populations isolées se trouvent dans les étangs de la zone minière.

Les stations qui possèdent des écrevisses présentent une plus grande altitude, une forte pente et des eaux plus froides et oxygénées que celles qui en sont dépourvues.

Les localités qui ont des écrevisses se situent dans une classe d'oxygénation moyenne à haute et on peut constater une nette séparation entre les stations les plus oxygénées qui conservent l'espèce autochtone A. pallipes et celles qui possèdent $P$. clarkii, avec un degré d'oxygénation plus bas.

Les analyses des spectres écologiques déterminés pour les deux systèmes ont montré que l'espèce présente une affinité pour l'eau plutôt froide, neutre (pH de 7,2 à 7,6) carbonatée (la dureté de l'eau est essentielle pour le développement de l'exosquelette) et qu'elle supporte des niveaux moyens d'eutrophisation.

Il nous semble qu'une récupération écologique des systèmes aquatiques soit indispensable, tout particulièrement dans certaines zones où, il n'y a pas longtemps, il existait encore de nombreuses populations d'écrevisses, notamment dans les secteurs moyen et supérieur de la rivière Cadagua. Une 
supérieur de la rivière Cadagua. Une protection spéciale des bassins ou des rivières qui possèdent encore des populations d'écrevisses autochtones, même réduites, comme dans les rivières Agüera, Mercadillo, Arratia, Lea et Artibai est souhaitable.

C'est sur ces bases que les organismes officiels doivent envisager la gestion et le contrôle des repeuplements.

\section{Travaux cités}

APHA-AWWA-WPCF, 1981. - Standar methods for the examination of water and wastewater. Whashington. Ed. : American Public Health Association. 1193 p.

BMDP Statistical Software. 1984. - BMDP Statistical Software, Celdde (fdD D, Gandiysof(Eafifortiesal (J.M.), Temiño (C.) \& Fernandez (R.). 1984. - Estado actual de las poblaciones y hábitats del cangrejo de rio (Austropotamobius pallipes Lereboullet) en la provincia de León. An. Fac. Vet. León, $30: 179-194$.

Celada (J.D.) \& Gaudioso (V.R.). 1985. - Localización y evaluación ecológica de las poblaciones y hábitats del cangrejo de río (Austropotamabius pallipes) en la provincia de León. Boletín de la Estación Central de Ecología, 27 : 99-105.

Celada (J.D.), Temiño (C.), Gaudioso (V.R.), Carral (J.M.) \& Fernandez (R.). 1986. - Repoblaciones astacicolas en la peninsula lbérica : consideraciones y líneas metodológicas generales. Jornadas de Estudio del cangrejo de río. Ed. Gobierno Vasco. Vitoria, Espa ıa (pp. 16-37).

Daguerre de Hurcaux (N.) \& Roqueplo (Ch.). 1981. - Définition du biotope préférentiel de l'écrevisse à pattes blanches, Austropotamobius pallipes (Ler_) dans un ruisseau landais. Bulletin Francais de Pisciculture, 281 : 211-222.
Docampo (L.) \& Rallo (A.). 1987. - Topologia de las comunidades de vertebrados (peces y anfibios) de la red hidrográfica de Vizcaya. II. Zonación de cuencas, estruct ura trófica de las estaciones y correlaciones de las especies con la altitud, pendiente y temperatura. Kobie. (XVI) : 269-29I.

G. de Bikuña (B.). 1987. - Importancia ecológica y bjogeográfica de los anfípodos e isópodos de las aguas superficiales de Bizkaia. Actes del Congreso de Biologia Ambiental (pp. : 137-148). Il. Congreso Mundial Vasco.

G. de Bikuña (B.), Basaguren (A.), Cacho (M.) \& Orive (E.). 1987. - Caracteristicas fisico-quimicas de las aguas superficiales de los principales ríos de Bizkaia. Actas del IV Congreso Español de Limnología (pp. : 165-177).

Golterman (H.L.). (ed.). 1969. - Meihods for chemical analysis of freshwoters. Oxford. IBP Handbook 8. Blackwell : $127 \mathrm{p}$.

Laurent (P.J.). 1980. - Utilisation des étangs pour la production d'écrevisses. In R. Billard, La Pisciculiure en étang. INRA. Publ. Paris : 333-342.

Orive (E.), Basaguren (A.), G. de Bikuña (B.) \& Cacho (M.). 1989. - A comparative study of water mineralization \& eutrophication in main water courses of Biscay, Basque Country. Water Research 23 (6) : 705-710.

Roqueplo (Ch.). 1983. - Etudes de populations naturelles d'écrevisses (Austropotamobius pallipes Ler.) dans le Sud-Ouest de la France. Etude P.A.M.R. ${ }^{\circ}$ 14. Groupe Daguerre de Hureaux (C.N.R.S.), 215 p.

Salazar (V.). 1983. - Peces y ríos de Alava. Vitoria-Gasteiz (Ed.), Diputación Foral de Alava, $134 \mathrm{p}$

Temiño (C.), Celada (J.D.) \& Carral (J.M.). 1986. - Estudio de las poblaciones astacicolas en los rios de la provincia de Burgos. Perspectivas. Jornadas de estudio del cangrejo de rio. Ed. Gobierno Vasco. Vitoria. España : 87-109.

Zariquiey (R.). 1968. - Crustáceos decápodos ibéricos. Barcelona. Ed. : C.S.1.C., 510 p. 